\title{
Erratum to: Effects of Three Types of Exercise Interventions on Healthy Old Adults' Gait Speed: A Systematic Review and Meta-Analysis
}

\author{
Tibor Hortobágyi ${ }^{1,2} \cdot$ Melanie Lesinski $^{3} \cdot$ Martijn Gäbler $^{1} \cdot$ Jessie M. VanSwearingen $^{4}$. \\ Davide Malatesta $^{5,6} \cdot$ Urs Granacher $^{3}$
}

Published online: 8 February 2016

(c) Springer International Publishing Switzerland 2016

\section{Erratum to: Sports Med (2015) 45:1627-1643 DOI 10.1007/s40279-015-0371-2}

Page 1630, column 2, section 2.3, paragraph 2, lines 15-20: The following sentence, which previously read:

To determine the effectiveness of an exercise intervention in relation to gait speed, we computed between-subject effect size (ES) using the implemented formula in Review Manager version 5.3 (Hedges' adjusted g) as (ES = $\pm[($ mean post-value intervention group) - (mean postvalue control group)]/pooled variance) [91].

The online version of the original article can be found under doi:10.1007/s40279-015-0371-2.

Tibor Hortobágyi

t.hortobagyi@umcg.nl

1 Center for Human Movement Sciences, University Medical Center Groningen, University of Groningen, A. Deusinglaan 1, 9700 AD Groningen, The Netherlands

2 Faculty of Health and Life Sciences, Northumbria University, Newcastle Upon Tyne, UK

3 Division of Training and Movement Sciences, University of Potsdam, Potsdam, Germany

4 Department of Physical Therapy, School of Health and Rehabilitation Sciences, University of Pittsburgh, Pittsburgh, PA, USA

5 Institute of Sport Sciences University of Lausanne (ISSUL), University of Lausanne, Lausanne, Switzerland

6 Department of Physiology, Faculty of Biology and Medicine, University of Lausanne, Lausanne, Switzerland

\section{Should read:}

To determine the effectiveness of an exercise intervention in relation to gait speed, we computed between-subject effect size (ES) using the implemented formula in Review Manager version 5.3 (Hedges' adjusted g) as (ES $=[$ (mean post-value intervention group) - (mean post-value control group)]/pooled standard deviation) [91]. 